\title{
DECISION SUPPORT SYSTEM FOR SOLVING THE STREET ROUTING PROBLEM
}

\author{
Peter Matis \\ Dept of Transportation Networks, University of Zilina, Univerzitná 8215/1, 01026 Zilina, Slovak Republic \\ E-mail:Peter.Matis@fria.utc.sk
}

Received 23 January 2008; accepted 12 June 2008

\begin{abstract}
Servicing a large number of customers in a city zone is often a considerable part of many logistics chains. The capacity of one delivery vehicle is limited, but, at the same time, it usually serves plenty of customers. This problem is often called a Street Routing Problem (SRP). Key differences between Vehicle Routing Problem (VRP) and SRP are presented here. The main problem of SRP is that when the number of customers is huge, the number of delivery path combinations becomes enormous. As the experimental results show in the case of SRP the error on the length of delivery routes based on an expert's judgment when compared to the optimal solution is in the range of $10-25 \%$. As presented in the paper, only using decision support systems such as Geographical Information Systems (GIS) makes possible to effectively manage SRP. Besides classical measurements used in VRP, such as total length of routes or time required for delivery in each route, other measurements, mostly qualitative ones, are presented. All of these are named as visual attractiveness. This paper discusses possible relationships between quantitative and qualitative measurements that give a promise for finding better solutions of SRP. Several new types of heuristics for solving SRP are evaluated and afterward compared using the real data. One of the key properties of GIS to use routing software is its flexible interactive and user-friendly environment. Routing software can find a good solution and explore the possibilities while an expert later can change the calculated routes to explore other possibilities based on the expert's judgment. This paper presents a practical use of new heuristics with the ArcView and solution of address mail for several cities in Slovakia served by Slovak Post ltd. Other Decision Support Systems that solve SRP are presented as TRANSCAD developed by Caliper Corporation or GeoRoute promoted by Canadian Post and GIRO.
\end{abstract}

Keywords: SRP, VRP, decision support system, GIS, heuristics, visual attractiveness.

\section{Introduction}

The Street Routing Problem (SRP), as a problem of servicing a large number of customers in a city zone, is often a part of many logistics chains (Amponsah and Salhi 2004). In the SRP category, we can include such problems as postal service delivery, commercial freight delivery, meter reading, newspaper delivery and waste removal.

Practical experience from the solutions of SRPs in the Slovak Republic suggests that in the majority of cases they are solved by an expert's experienced judgment. The main problem of SRP is that the number of customers is large and the number of delivery path combinations is enormous. As the experimental results show in the case of SRP the error on the length of delivery routes based on the expert's judgment when compared to optimal solution is in the range of $10-25 \%$.

SRP is in many cases similar to the classical Vehicle Routing Problem (VRP). There are some important differences between SRP and VRP.
- If compared to VRP, the number of customers in SRP is larger and can be expressed in hundreds of thousand, whereas in VRP the number of customers is only in hundreds.

- The service time for one customer in SRP is short and the route path for one vehicle contains several hundreds of these customers.

- The density of the street network in SRP is high compared to that in VRP.

- The distance between two close customers in SRP is small and therefore a possibility of getting from one customer to another through a small number of possible network connections exists.

- Traffic regulations in the city zone are more complicated and more restrictive than traffic regulations in a regular road network. These regulations can and do greatly affect the expense for the routes.

SRP requires large datasets including the city street networks, their parameters and regulations as well as the positions of customers with all their attributes and con- 
nection to the street network. All of this is possible in the Geographical Information System (GIS). Connecting GIS, user interface and solution algorithms we can create a decision support system (DSS) for the management of SRP (Ruiz et al. 2004; Silva et al. 2006).

\section{Experimental sample}

Unlike VRP, SRP is a less explored group of problems and is not a good, publicly available sample of data for method comparison. Some of the authors, for example (Amponsah and Salhi 2004) usually solve specific problems, and thus it is difficult to compare solutions across these problems because each of those is somewhat unique.

It is necessary to use our own experimental sample covering most of the typical SRP. Simultaneously, the possibility of comparing each of solution methods arises. Our experimental sample embraced several cities and large villages in the Slovak Republic. The customers were houses in these cities where full street infrastructure was available.

Data was manually collected from the source maps ZM 1:10 000 purchased from Geodetic and Cartographic Institute of the Slovak Republic. The total number of customers in the sample is 101977 and the total number of settlements is 90, averaging 1133 customers per settlement, which is more than the average number of houses in the cities and villages in the whole Slovak Republic.

The customers in SRP usually are each house or door in blocks. They are typically located on both sides of the street segments and could be serviced differently depending on local conditions and other regulations. Fig. 1 shows three typical cases.

\section{Customer aggregation}

Practical experiments show that if we want to apply VRP heuristics to the solution of SRP, we need to aggregate customers to be able to solve the problem (Matis 2006). One of the purposes of dealing with this issue is the fact

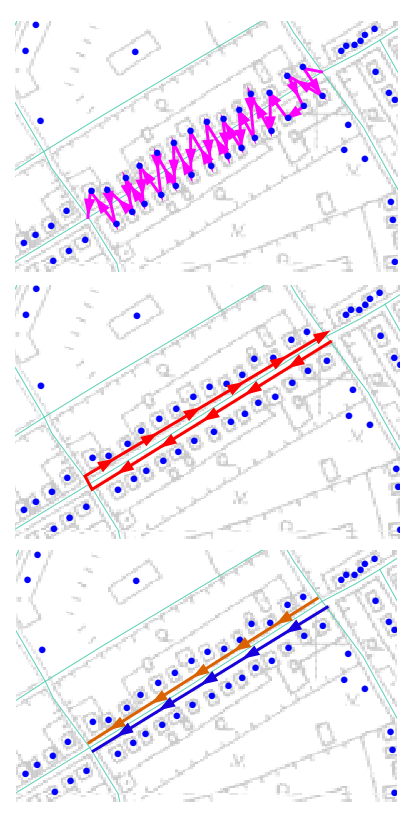

Fig. 1. Cases of service on the street segments that computational time expands with large numbers of customers and, by aggregation, we keep this number in a range that is comparable to the typical VRP:

- In distance aggregation, we aggregate customers based on their distance. It is possible to aggregate customers located on different street segment(s).

- Line aggregation only allows the aggregation of customers located on the same side of a single street segment.

- Line-segment aggregation is a special case of line aggregation where all customers on one side of one street segment create one aggregate.

- Address aggregation allows the combination of customers having similar addresses.

Experiments have shown that the best aggregation is distance aggregation. On the other hand, distance aggregation shows high deformation of aggregated positions against the original positions of customers.

\section{Visual attractiveness of routes}

The visual attractiveness of routes is of great importance in practical routing applications and plays a central role in whether or not the routes are adopted. As an example, in many business and other enterprises, service personnel (drivers) must serve geographically dispersed customers. The service region is divided into districts and each driver is responsible for their own district. These districts typically do not overlap. As such, the drivers and their managers often notice that the trips developed by algorithms to solve SRP may cross one another and are consequently reluctant to employ the resulting trips. The drivers believe that the trips must be inefficient if they cross. Since the existing techniques do not explicitly consider solution shape, the resulting trips are difficult to implement in practice.

Poot et al. (2002) describe some possible parameters that could be used for measurement. The properties of compactness, crossing and distance from cluster median are the parameters that are hard to measure and are as important as other operational constraints. Therefore, they are often treated as soft constraints. There is no publication describing these measurements in detail. We have defined these measurements based on some experiments in the following way (Matis 2006).

A route is considered to be compact if all customers in the route are within short distances of one another. One can measure compactness of route $i$ :

$$
\mathrm{COMP}_{i}=\frac{\text { AvgDist }_{i}}{\text { AvgMaxDist }_{i}},
$$

where $A v g D i s t_{i}$ is an average distance of two immediate following customers in the route $i$, and AvgMaxDist $t_{i}$ is an average distance $20 \%$ of the longest distances between two immediate customers in the route $i$.

The service region is divided into districts and the drivers are responsible for their own districts. These districts typically do not overlap. As such, the drivers and their managers who notice that routes developed by al- 
gorithms may cross one another are reluctant to employ the resulting routes.

The measure $D G R B$ gives the average number of customers in a route which is closer to the center of gravity of another route than to the center of gravity of the route itself. Here, the center of gravity of a route is the center of gravity calculated from the coordinates of the locations of the customers in the route. We can measure it as:

$$
D_{G R B_{i}}=2 \cdot\left(1-\frac{\left|\hat{O}_{i}\right|}{\left|O_{i}\right|}\right)-1,
$$

where $\left|\hat{O}_{i}\right|$ is a number of customers closer to the center of gravity of another trip, and $\left|O_{i}\right|$ is a number of all customers in the route $i$.

We can measure visual attractiveness as:

$$
V A_{i}=\frac{1}{\frac{N C_{i}}{5}+\frac{1}{D G R B_{i}}+\frac{1}{C O M P_{i}}-1},
$$

where $N C_{i}$ represents the number of crossings of route $i$ with other routes in the service part of the route. We do not count crossings that are between the depot and first customers of the route and the last customers on the route and the depot. The reason for equation (3) is to get visual attractiveness as a number in the range $<0.1>$. The higher values of visual attractiveness are usually more acceptable. We have to remember that in real life, customers are distributed randomly and street networks do not have compact density. In most of the real cases, we cannot get visual attractiveness measurement higher than 0.5.

Solutions of SRP for the Klenovec are shown in Figs 2 and 3.

\section{New heuristics for solution of SRP}

There is no heuristics that specializes in SRP and also use criteria other than quantitative measurements. We have tried to implement and compare nine new types of heuristics (Matis 2007).

The types of heuristics are as follows:

- Heuristics uses primary aggregation: cluster-first, route-second methodology. The goal is to create 'natural clusters' and then run several traveling salesman problems - one on each cluster.

$\triangleright$ Greedy algorithm - Unified Cluster First Route Second (UCFRS) first estimates the number of clusters using some other heuristics (for example, Yellow). Then, this heuristics places the centers of clusters solving the p-median problem (Zhao and Batta 1999) It evaluates customers in order of values

$v_{i}=\min _{t_{j}}-\min _{t_{k}}$,

where: $\min _{t_{k}}$ is the road distance from customer $i$ to the closest center and $\min _{t_{j}}$ is the road distance to the second closest center. First, take the customer $i$ with the maximum

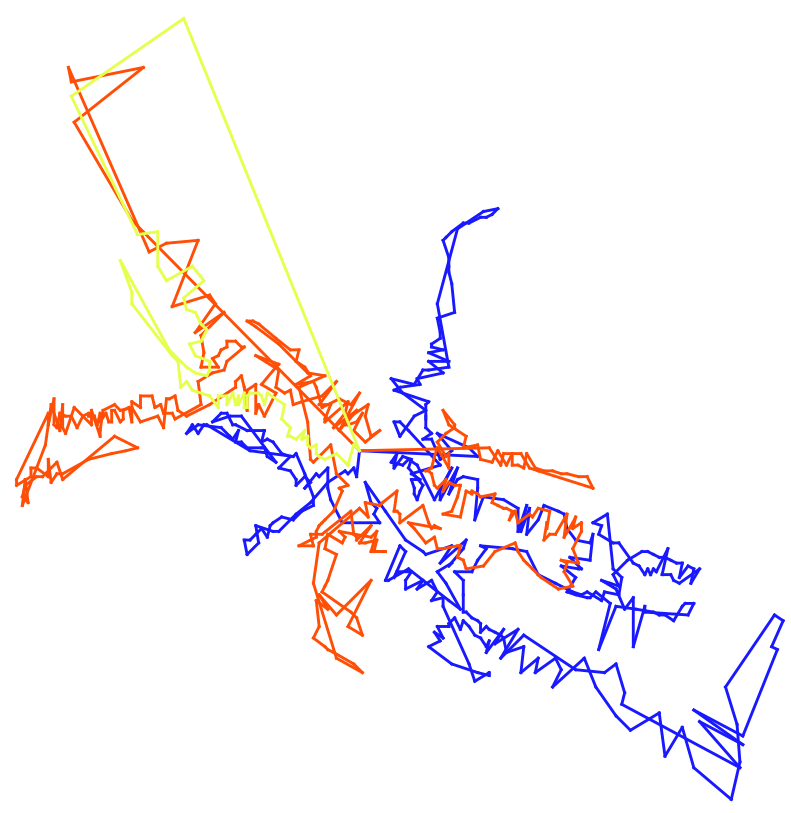

Fig. 2. Solutions of SRP for the Klenovec with the total length $=62209 \mathrm{~m}$ and visual attractiveness $=0.075$

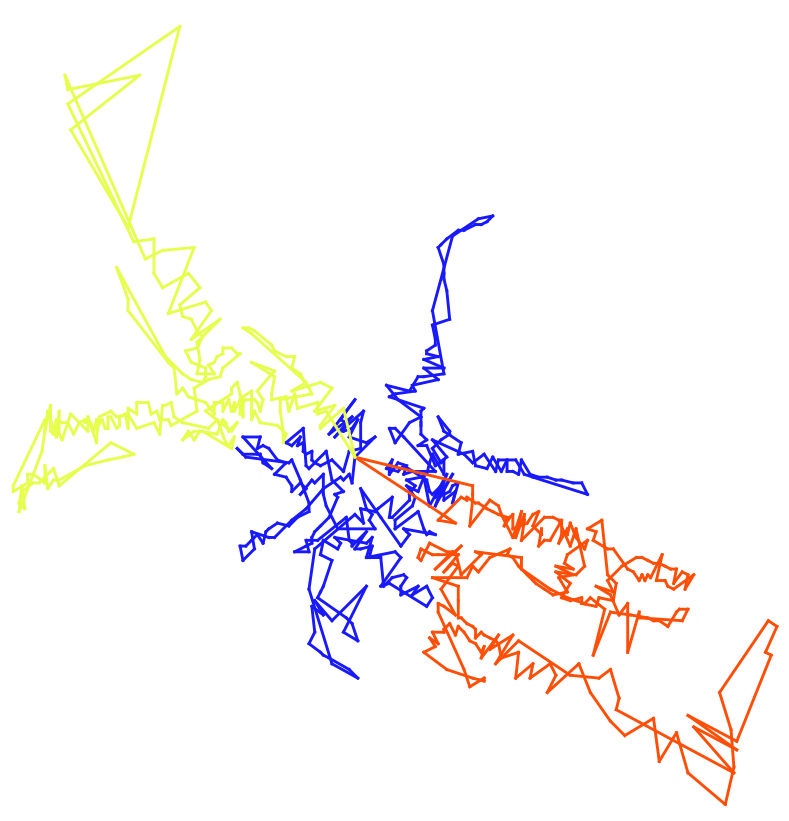

Fig. 3. Solutions of SRP for the Klenovec with the total length $=63553 \mathrm{~m}$ and visual attractiveness $=0.461$

value of $v_{i}$. In the evaluation, it assigns customers to the centers based on their road distance to the centers and the cluster limits (time, weight, number of customers etc.).

$\triangleright$ Revised greedy algorithm (UCFRS2) is similar to the previous heuristics. The difference is that customers are assigned to the $p$-centers based on the road distance, regardless of cluster limits. If some clusters are over the limits, they are balanced. It uses a special transportation method algorithm for balancing. 
- Shaping heuristics

$\triangleright$ Two Steps Savings Heuristics (TSSH) in the first step creates the clusters using UCFRS2 heuristics. In the second step, it is similar to the Yellow heuristics. Savings are calculated as

$v_{i j}=c_{S i}+c_{j S}-(\gamma+\lambda \cdot z \cdot(i, j)) \cdot c_{i j}$,

where: $y$ is the shaping parameter like in the Yellow heuristics, $\lambda$ is a new shaping parameter and $z(i, j)$ is a function returning 0 if customers $i$ and $j$ belong to the same cluster and 1 otherwise, $c_{i j}, c_{S i}$ are the road distance between customers $i$ and $j$ or depot and customer $i$.

$\triangleright$ Revised Two Steps Savings Heuristics (RTSSH) uses savings calculated as

$v_{i j}=c_{S i}+c_{j S}-\left(\gamma+\lambda_{0}\right) \cdot c_{i j} ; \quad i \in I ; j \in I$

$v_{i j}=c_{S i}+c_{j S}-\left(\gamma+\lambda_{1}\right) \cdot c_{i j} ; \quad i \in I ; j \notin I$,

where $\lambda_{0}, \lambda_{1}$ are new shaping parameters.

- Metaheuristics

$\triangleright$ Tabu search for SRP (TABU) starts with a solution created by RTSSH. It takes one customer from one route and tries to attach him/her to the other route without reordering customers in either route. It also takes the solutions worse than the best existing solution or the solution exceeding capacity limits in order to go out of the local minimum.

- Heuristics using visual attractiveness

$\triangleright$ Revised Yellow heuristics (RYellow) uses savings based on the calculations of partial visual attractiveness when two routes are merged to one route:

$v_{j p}=c_{S j}+c_{p S}-\left(\gamma+\mu \cdot\left(1-Z V A_{j p}\right)\right) \cdot c_{j p}$,

where: $\mu$ is a new shaping parameter, $Z V A_{j p} \in$ $<0.1>$ represents a change in the visual attractiveness if the routes where customers $j$ and $p$ are located are merged to one route. Visual attractiveness calculated in this method uses only compactness and GRB.

$\triangleright$ Two Steps Savings Heuristics with Visual Attractiveness (TSSH-VA) works in the same way as TSSH only after generating each solution and tries to find the minimum of function in (8) instead of simple minimum cost or minimum length.

$\min U F V=w \cdot V A+(1-w) \cdot \frac{U F}{U F_{\text {Yellow }}}$,

where: $w$ is the weight of visual attractiveness, $U F$ is a value of objective function (total cost, total length...), and $U F_{\text {yellow }}$ is a value of objective function for a solution given by Yellow heuristic.

- Heuristics with fuzzy clustering

$\triangleright$ Fuzzy Cluster Heuristics (FCH) is a complicated heuristics as it uses clustering by 'fuzzy c-means' (FCM). The membership of custom- ers to each cluster is set as a triangle fuzzy number. It is similar to TSSH heuristics.

- Heuristics with a mixed model of node and arc service

$\triangleright$ TSSH that use NEARP representation of graph is revised TSSH. In this variation of TSSH, the NEARP representation of graph is used (Lacomme et al. 2003). In this case, some customers on the street segments are aggregated and a street segment is viewed as a customer. We get a mixture graph where some customers are nodes and some are arcs.

We evaluated the performance of each heuristics on the experimental sample data. Table 1 summarizes the results of the experiments.

Table 1. Parameters of new SRP heuristics

\begin{tabular}{lccc}
\hline \multicolumn{1}{c}{ Heuristics } & ALY & VA & TIME \\
\hline Sweep & 75 & 0.29 & 7.5 \\
\hline Yellow & 0 & 0.35 & 125.7 \\
\hline UCFRS & 16 & 0.28 & 11.5 \\
\hline UCFRS2 & 10 & 0.40 & 21.3 \\
\hline TSSH & -3 & 0.38 & 228.5 \\
\hline RTSSH & -3.6 & 0.38 & 453.2 \\
\hline TABU & -4.0 & 0.39 & 12652.3 \\
\hline RYellow & -1.5 & 0.42 & 10468.2 \\
\hline TSSH-VA & -2.2 & 0.44 & 447.3 \\
\hline FCH & -1.4 & 0.39 & 212.1 \\
\hline TSSH-NEARP & -0.4 & 0.34 & 12.4 \\
\hline
\end{tabular}

ALY - average relative total length compared to solution by Yellow heuristics in\%, VA - average visual attractiveness, TIME - average computing time in seconds

We can see that we did not create one superior heuristics that could be used for all SRP cases. We offer a choice in the decision support system for the SRP where the user specifies available time for a calculation and desired parameters of results and the system chooses the best type of heuristics.

\section{Decision support system for the SRP}

The major obstacle that prevents mathematical models and heuristics from being practically used for solving SRP in Slovakia is the nonexistence of tools and methods for supporting SRP solution. A decision support system (tool) should meet the following requirements:

- It is important that the tool visualizes results and they are visually controllable.

- Difference in data and SRP specifics requires different solving methods for each case. The user should be able to choose the best method for his needs or the choice should be automatic.

- The tool must allow interactive changes in the results and the evaluation of these changes.

- The tool may allow simulations to check the solution. 
There are several applications containing tools for reaching a solution to transportation problems. Some of those could be used for SRP. For example:

- GeoRoute by GIRO represents an independent application solution for the most part of SRP. It is a closed system and the user cannot choose the methods for a solution as it is an expensive solution.

- ArcGIS Network Analyst is an extension of the standard GIS system by ESRI. It is an open system and provides a solution for VRP. The types of heuristics used for the solution are very limited and very slow.

- TRANSCAD by Caliper is a specialized GIS for transportation planning and solving transportation problems and includes heuristics for solving VRP and ARP but not heuristics for SRP.

- GeoMap by GeoMap is an independent application having GIS and CAD attributes. It is a closed system for solving VRP.

An important feature of the decision support system is good visualization and a good editor. The tools able to visualize results are easily acceptable to users. Visualization also allows users to see any problems or discrepancies which are not easy to find or implement by heuristics. The user can change starting conditions in the problem to avoid these discrepancies, test a new model and get an acceptable result by several iterations.

To shorten development time, we have chosen a standard GIS environment as the base system for the data management. Fig. 4 shows the connections of all components in the decision support system for solving SRP.

The integrator represents the main user interface that takes control over each type of heuristics - a connection of GIS database to heuristics or to an interpreter. It is used for model creation and management. We used ArcView by ESRI as the main GIS tool because it is an open system that allows the user to program its functionalities. GIS is managed from the integrator by the Avenue scripting language, $\mathrm{C} \#$, and VBasic depending on the version of ArcView. GIS is used in the system as a data management tool and editor for visualizing and editing results.

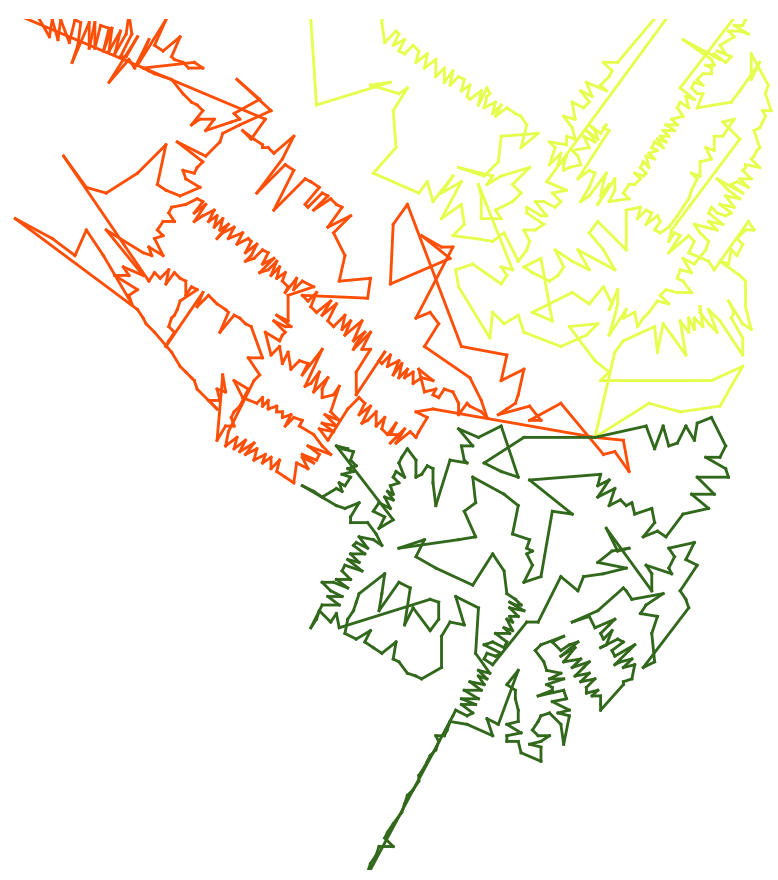

Fig. 4. Routes in Malacky from TSSH heuristics

The module for heuristics also includes a system for SRP model management and algorithms for evaluating quantitative and qualitative parameters of solutions.

The interpreter is a supplemental module that helps one to visualize other parameters of SRP hardly visualized in the GIS environment.

For simplicity, we expect that the GIS database for each SRP case will have a unified form. Data is stored in the natural GIS database - in this case - an ESRI shapefile format and relational database. Figs 5 and 6 documents the elements of the GIS database and relations.

\section{Conclusions}

1. There are expanding activities in the cities that can be presented as street-based tasks. We introduced some methodology for solving general SRPs. One of the possible solutions for dealing with this problem is to

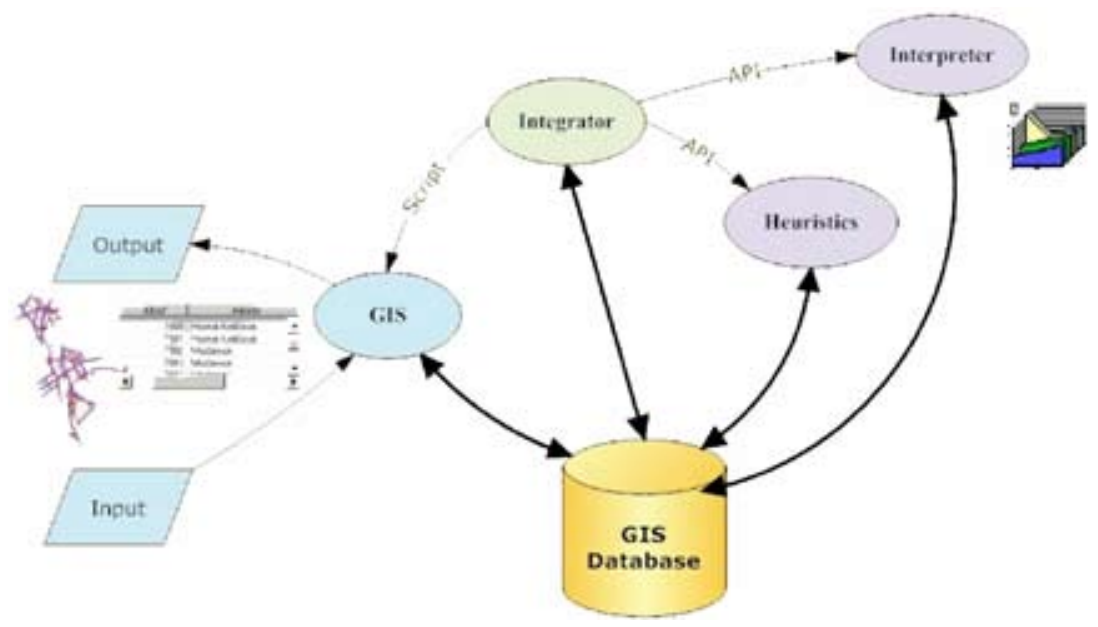

Fig. 5. Decision support system for SRP 


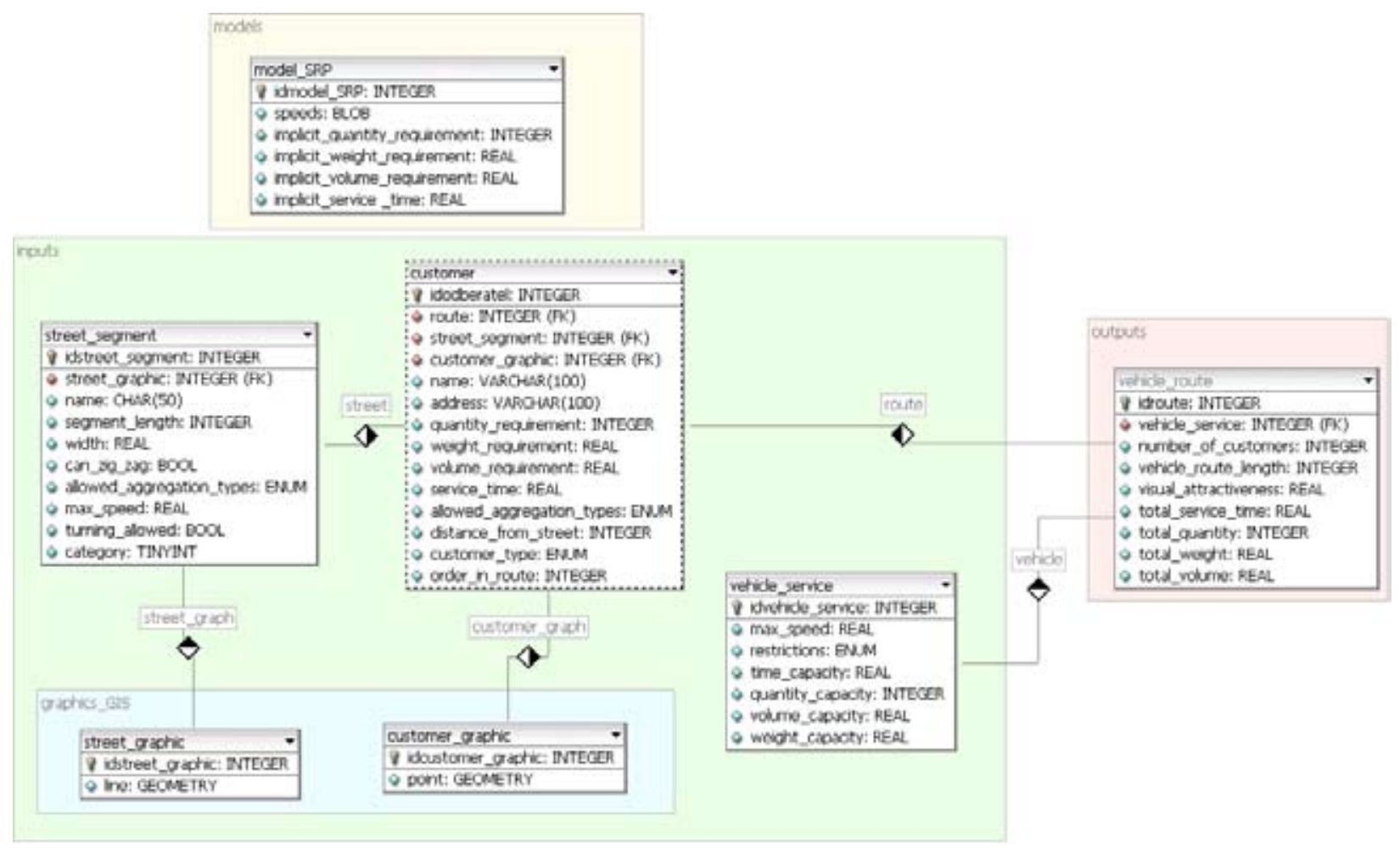

Fig. 6. GIS database for SRP

aggregate a large number of customers into clusters and then solve VRP. The conducted experiments disclosed that other types of heuristics were needed for solving SRP.

2. The criteria other than the usual measurements are needed to evaluate the results. In particular, visual attractiveness needs to be added as a non-standard quality measurement for resulting routes. There is some relation between visual attractiveness and the quality of resulting routes.

3. This paper presents 9 new types of heuristics for SRP and their promising results. Our analysis indicates that there is not a single type of heuristics able to solve any SRP case. Instead, a combination of heuristics is needed to solve a specific case.

4. DSS for SRP can address this shortfall. We presented DSS based on the GIS database. We used DSS in 5 Slovakian cities. Having applied the solutions, we were able to shorten the total length of all tours up to $10 \%$ in each case.

\section{References}

Amponsah, S. K.; Salhi, S. 2004. The investigation of a class of capacitated arc routing problems: the collection of garbage in developing countries, Waste Management 24(7): 711- 721 .

Lacomme, P.; Prins, Ch.; Ramdane-Cherif, W. 2003. Evolutionary algorithms for arc routing problems, in Third Meeting of the Spanish research project SADERYL. Conference on Routing and Location CORAL, Tenerife, Spain. Available from Internet: <http://webpages.ull.es/users/saderyl/nold/Contenido/abstracts/Prins.pdf $>$.
Matis, P. 2006. Management of street routing problems using decisions support system, Communications, Zilinska univerzita $v$ Ziline 3: 5-8.

Matis, P. 2007. The relationship between quantitative and qualitative measurements in solving of street routing problems, in $15^{\text {th }}$ International Scientific Conference on Mathematical Methods in Economics and Industry, Herlany 2007, 144152.

Poot, A; Kant, G.; Wagelmans, A. 2002. A saving based method for real-life vehicle routing problems, Journal of the Operational Research Society 53(1): 57-68.

Ruiz, R.; Maroto, C.; Alcaraz, J. 2004. A decision support system for a real vehicle routing problem, European Journal of Operational Research 153(3): 593-606.

Silva, C G.; Figueira, J.; Lisboa, J.; Barman, S. 2006. An interactive decision support system for an aggregate production planning model based on multiple criteria mixed integer linear programming, Omega 34(2): 167-177.

Zhao, P.; Batta, R. 1999. Analysis of centroid aggregation for the Euclidean distance $p$-median problem, European Journal of Operational Research 113(1): 147-168. 